\title{
POLAR QUOTIENTS OF A PLANE CURVE AND THE NEWTON ALGORITHM
}

\author{
ANDRZEJ LENARCIK \\ Dedicated to Professor Arkadiusz Płoski
}

\begin{abstract}
Using the Newton algorithm we show how to compute all the polar quotients and their multiplicities of a plane curve $f=0$, where $f$ is a formal power series of two variables over an algebraically closed field $\mathbf{k}$ with characteristic zero. The curve is not necessarily reduced.
\end{abstract}

\section{Introduction}

Let $\mathbf{k}$ be an algebraically closed field with characteristic zero. We use standard notations; $\mathbf{k}[[X, Y]]$ is the ring of formal power series, ord $f$ is the order of $f \in \mathbf{k}[[X, Y]] \quad($ ord $0=+\infty)$. For elements $a_{1}, \ldots, a_{p}$ of a given set we define the system $\mathscr{A}=\left\langle a_{1}, \ldots, a_{p}\right\rangle$ as the sequence $a_{1}, \ldots, a_{p}$ treated as unordered. Put $\operatorname{deg} \mathscr{A}=p$. Instead of $\langle\underbrace{a_{1}, \ldots, a_{1}}_{m_{1} \text { times }}, \ldots \underbrace{a_{p}, \ldots, a_{p}}_{m_{p} \text { times }}\rangle$ we write $\left\langle a_{1}: m_{1}, \ldots a_{p}: m_{p}\right\rangle$. For $\mathscr{A}=\left\langle a_{1}, \ldots, a_{p}\right\rangle$ and $\mathscr{B}=\left\langle b_{1}, \ldots, b_{q}\right\rangle$ we have a natural addition $\mathscr{A} \oplus \mathscr{B}=$ $\left\langle a_{1}, \ldots a_{p}, b_{1}, \ldots b_{q}\right\rangle$ with the neutral element \langle\rangle . By convention $\langle a: 0\rangle=\langle\rangle$. (see [Wh], notion of symetric power).

Let $f(X, Y) \in \mathbf{k}[[X, Y]]$ be such a series that $p=$ ord $f(0, Y)>1$. Recall the polar curve $\partial f / \partial Y=0$ with its positive-order-roots $z_{1}(X), \ldots, z_{p-1}(X)$ in the ring $\mathbf{k}[[X]]^{*}=\bigcup_{n \geq 1} \mathbf{k}\left[\left[X^{1 / n}\right]\right]$ of the Puiseux series. We consider the system

$$
\overline{\mathcal{Q}}(f, X)=\left\langle\text { ord } f\left(X, z_{1}(X)\right), \ldots, \text { ord } f\left(X, z_{p-1}(X)\right)\right\rangle
$$

of polar quotients of $f$ with respect to $X$. Every polar quotient is either a positive rational or $+\infty$. We omit the bar over 2 to denote the system of finite quotients. We have $\overline{2}(f, X)=\mathscr{2}(f, X)$ if and only if $f$ is reduced.

2000 Mathematics Subject Classification: Primary 32S55.

Key words and phrases: plane curve singularity, polar quotients, Newton polygon, Newton algorithm.

Supported in part by the KBN grant No 2 P03A 02215.

Received February 25, 2004; revised July 23, 2004. 
When $X$ and $f$ are transverse, the polar quotients are topological invariants called polar invariants $(\mathbf{k}=\mathbf{C})$. We have $[\mathrm{T}]$ :

$($ Lojasiewicz exponent of $\operatorname{grad} f$ near zero $)=($ maximal polar invariant $)-1$.

The study of polar quotients and polar invariants extends over many authors ([T], [M], [D], [E], [Eph], [CA], [LMW1], [LMW2], [Ga], [GP1], [GP2], [LMP]). Let us mention Merle's $[\mathrm{M}]$ description of polar invariants for irreducible series (see also [GP1]); Delgado's developement of the case of two branches [D]; and the computation of polar invariants for multi-branched singularity using Egger's diagrams $([\mathrm{E}],[\mathrm{Ga}])$. The authors of [GP2] give explicit formulae for the polar quotients in terms of characteristics and intersection multiplicities of branches. For a nondegenerate series $f$ the polar invariants can be calculated using the Newton polygon of $f[\mathrm{LP}]$, [LMP].

This paper aims to compute the system of polar quotients using a version of the Newton algorithm ([W], [Can], [KP], [L2]). We generalize the approach from $[\mathrm{LP}],[\mathrm{LMP}]$.

\section{Main result}

It is convenient to consider the ring $\mathbf{k}\left[\left[X^{*}, Y\right]\right]=\bigcup_{n \geq 1} \mathbf{k}\left[\left[X^{1 / n}, Y\right]\right]$. Take $f=\sum f_{\alpha \beta} X^{\alpha} Y^{\beta} \in k\left[\left[X^{*}, Y\right]\right]$. As usual we define the support supp $f$ as $\{(\alpha, \beta)$ : $\left.f_{\alpha \beta} \neq 0\right\}$, the Newton diagram $\Delta(f)$ as $\operatorname{conv}\left(\operatorname{supp} f+\mathbf{R}_{+}^{2}\right)$, and the Newton polygon $\mathscr{N}(f)$ as the set of compact faces of $\Delta(f)$. By $\delta(f)$ we denote the distance between $\Delta(f)$ and the horizontal axis.

For $S \in \mathscr{N}(f)$, by $|S|_{1}$ and $|S|_{2}$ we denote the lengths of projections of $S$ onto the horizontal and vertical axies, respectively. We call the ratio $|S|_{1} /|S|_{2}$ the inclination of $S$. For $\theta>0$ (or $\theta=-\infty$ ) it will be useful to consider the polygon $\mathscr{N}^{\theta}(f)$, which consists of all the faces $S \in \mathscr{N}(f)$ with an inclination strictly greater than $\theta$. Let $\alpha(S)$ denote the abscissa of the point where the line determined by $S$ intersects the horizontal axis. We define the initial form $\operatorname{in}(f, S)=\sum f_{\alpha \beta} X^{\alpha} Y^{\beta}$, where $(\alpha, \beta)$ runs over $S \cap \operatorname{supp} f$. By $t(f, S)$ we denote the number of different roots of the polynomial in $(f, S)(1, Y) \in k[Y]$. The number $\varepsilon(S) \in\{-1,0\}$ is defined as -1 when $S$ touches the horizontal axis and as 0 otherwise. Put $d(f, S)=|S|_{2}+\varepsilon(S)-t(f, S)+1$. Note that $d(f, S)=0$ if and only if every nonzero root of $\operatorname{in}(f, S)$ in $\mathbf{k}[[X]]^{*}$ is of multiplicity 1 . Then we call the series $f$ nondegenerate on $S$.

For any $\varphi \in \mathbf{k}[[X]]^{*}$, ord $\varphi>0$ one can apply the substitution $f_{\varphi}(X, Y)=$ $f(X, \varphi(X)+Y) \in \mathbf{k}\left[\left[X^{*}, Y\right]\right]$ ([Can], [GP1], [KP $]$ ). Clearly, $f_{\varphi}=f$ for $\varphi=0$. Consider the ring $\mathbf{k}[X]^{*}=\bigcup_{n \geq 1} \mathbf{k}\left[X^{1 / n}\right]$ of Puiseux polynomials. For $\varphi \in \mathbf{k}[X]^{*}$, $\operatorname{deg} \varphi<+\infty$. Put deg $0=-\infty$. The set $T(f, X) \subset \mathbf{k}[X]^{*}$ of the tracks (of the Newton algorithm) for $f$ is defined to be the minimal set satisfying two properties: (I) $0 \in T(f, X)$, (II) for every $\varphi(X) \in T(f, X)$, if there exists $S \in \mathscr{N}^{\operatorname{deg} \varphi}\left(f_{\varphi}\right)$, then for every nonzero root $a X^{\theta}$ of $\operatorname{in}\left(f_{\varphi}, S\right), \varphi+a X^{\theta} \in T(f, X)$. We will write $\mathscr{N}_{\varphi}$ instead of $\mathscr{N}^{\operatorname{deg} \varphi}\left(f_{\varphi}\right)$ when $f$ is fixed. 
We call series $\psi \in \mathbf{k}[[X]]^{*}$ a continuation of $\varphi \in \mathbf{k}[X]^{*}$ if $\operatorname{ord}(\varphi-\psi)>\operatorname{deg} \varphi$. Then we write $\psi=\varphi+\cdots$. Let $\varphi$ be a track of the Newton algorithm for $f$. Let $\overline{2}_{\varphi}(f, X)=\bigoplus\langle$ ord $f(X, z(X))\rangle$, where $z(X)$ is a continuation of $\varphi$. By analogy we define $\mathscr{Q}_{\varphi}(f, X) \subset \mathscr{Q}(f, X)$ for ord $f(X, z(X))<+\infty$.

Now, put $\mathscr{C}_{\varphi}=\left\langle\infty: \delta\left(f_{\varphi}\right)-1\right\rangle$ if $\delta\left(f_{\varphi}\right)>1$ and $\mathscr{C}_{\varphi}=\langle\rangle$ if $\delta\left(f_{\varphi}\right)=1$ or 0 . For $S \in \mathscr{N}_{\varphi}$ we denote $\mathscr{A}_{\varphi, S}=\left\langle\alpha(S): t\left(f_{\varphi}, S\right)-1\right\rangle$. We have the following

THEOREM 2.1 (main result).

(a) For $\varphi \in T(f, X)$ we have $\overline{\mathscr{Q}}_{\varphi}(f, X)=\left[\bigoplus_{S \in \mathscr{N}_{\varphi}}\left(\mathscr{A}_{\varphi, S} \oplus \mathscr{B}_{\varphi, S}\right)\right] \oplus \mathscr{C}_{\varphi}$, where $\mathscr{B}_{\varphi, S}$ is a system of quotients strictly greater than $\alpha(S)$, deg $\mathscr{B}_{\varphi, S}=$ $d(f, S)$.

(b) $\mathscr{B}_{\varphi, S}=\bigoplus_{a X^{\theta}} \overline{\mathscr{Q}}_{\varphi+a X^{\theta}}(f, X)$, where aX $X^{\theta}$ runs over all multiple nonzero roots of $\operatorname{in}\left(f_{\varphi}, S\right)$,

(c) $2(f, X)=\bigoplus_{\varphi \in T(f, X)} \bigoplus_{S \in \mathcal{N}_{\varphi}} \mathscr{A}_{\varphi, S}$

We prove the above theorem in the next section.

Remark 2.2. Clearly $\alpha(S)$ is a polar quotients if and only if $t\left(f_{\varphi}, S\right)>1$. This condition is always satisfied for $S \in \mathscr{N}_{\varphi}$, which does not touch the horizontal axis. When $\alpha(S)$ is not a polar quotient then $S$ touches the axis and $\operatorname{in}\left(f_{\varphi}, S\right)=$ $b X^{\zeta}\left(Y-a X^{\theta}\right)^{|S|_{2}}$.

Remark 2.3. We can consider the system $\mathscr{A}_{\varphi}(f, X)=\bigoplus_{S \in \mathcal{N}^{\varphi}} \mathscr{A}_{\varphi, S}$ of finite quotients which are determined by the behaviour of $f_{\varphi}$ on $\mathscr{N}_{\varphi}$. Clearly $\operatorname{deg} \mathscr{A}_{\varphi}(f, X)=\sum_{S \in \mathcal{N}^{\varphi}}\left[t\left(f_{\varphi}, S\right)-1\right]$.

Remark 2.4. We state that the sum in (c) is in fact finite. We can replace $T(f, X)$ by the finite $T_{\min }(f, X)$, which contains all $\varphi \in T(f, X)$ such that there exists $S \in \mathscr{N}_{\varphi}$ with $t\left(f_{\varphi}, S\right)>1$.

For $\varphi=0$ we have the following two corollaries. We write $\mathscr{A}_{S}, \mathscr{B}_{S}$ instead of $\mathscr{A}_{0, S}, \mathscr{B}_{0, S}$ and $\mathscr{C}$ instead of $\mathscr{C}_{0}$.

Corollary 2.5. (a) $\overline{\mathscr{Z}}(f, X)=\left[\bigoplus_{S \in \mathscr{N}(f)}\left(\mathscr{A}_{S} \oplus \mathscr{B}_{S}\right)\right] \oplus \mathscr{C}$,

(b) $\mathscr{B}_{S}=\bigoplus_{a X^{\theta}} \overline{\mathscr{Q}}_{a X^{\theta}}(f, X)$, where a $X^{\theta}$ runs over all possible multiple nonzero roots of in $(f, S)$.

Corollary 2.6. If $\delta(f) \leq 1$ and $d(f, S)=0$ for every $S \in \mathscr{N}(f)$ then

$$
\overline{\mathscr{Q}}(f, X)=\mathscr{Q}(f, X)=\mathscr{A}_{0}(f, X)=\underset{S \in \mathscr{N}(f)}{\bigoplus}\left\langle\alpha(S):|S|_{2}+\varepsilon(S)\right\rangle .
$$




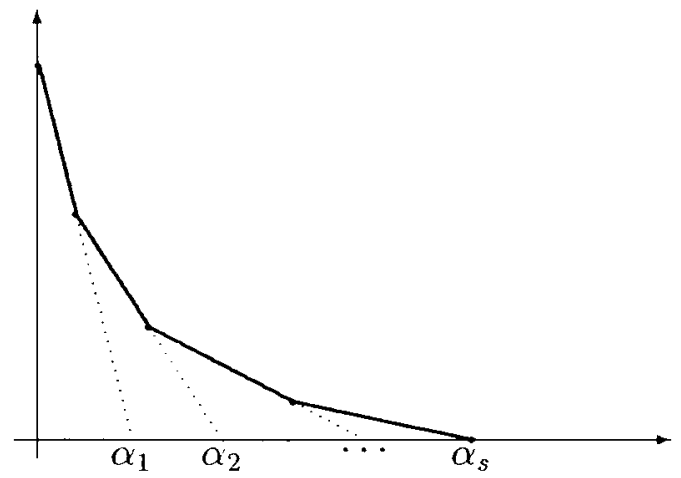

When $S \in \mathscr{N}(f)$ touches the horizontal axis and $|S|_{2}=1$ then $\alpha(S)$ is not a polar quotient. We call such a face exceptional ([LP]).

EXAmple 2.7. Consider the curve $f=Y\left(Y^{2}-X\right)^{3}-2 X Y^{2}\left(Y^{2}-X\right)^{2}+$ $8 X^{5} Y+X^{6}$. We have $\mathscr{N}(f)=\{S, T\}$, where $S$ joins $(0,7)$ with $(3,1)$ and $T$ joins $(3,1)$ with $(6,0)$. From Corollary $2.5 \overline{2}(f, X)=\mathscr{A}_{S} \oplus \mathscr{B}_{S} \oplus \mathscr{A}_{T} \oplus \mathscr{B}_{T} \oplus$ $\mathscr{C}$. Clearly $\operatorname{in}(f, S)=Y\left(Y^{2}-X\right)^{3}=Y\left(Y-X^{1 / 2}\right)^{3}\left(Y+X^{1 / 2}\right)^{3}, \quad \alpha(S)=7 / 2$, $t(f, S)=3, \quad d(f, S)=4 ; \quad \operatorname{in}(f, T)=-X^{3} Y+X^{6}=-X^{3}\left(Y-X^{3}\right), \quad \alpha(T)=6$, $t(f, T)=1, d(f, T)=0$ and $\delta(f)=0$. Obviously $\mathscr{A}_{T}=\mathscr{B}_{T}=\mathscr{C}=\langle\rangle$.
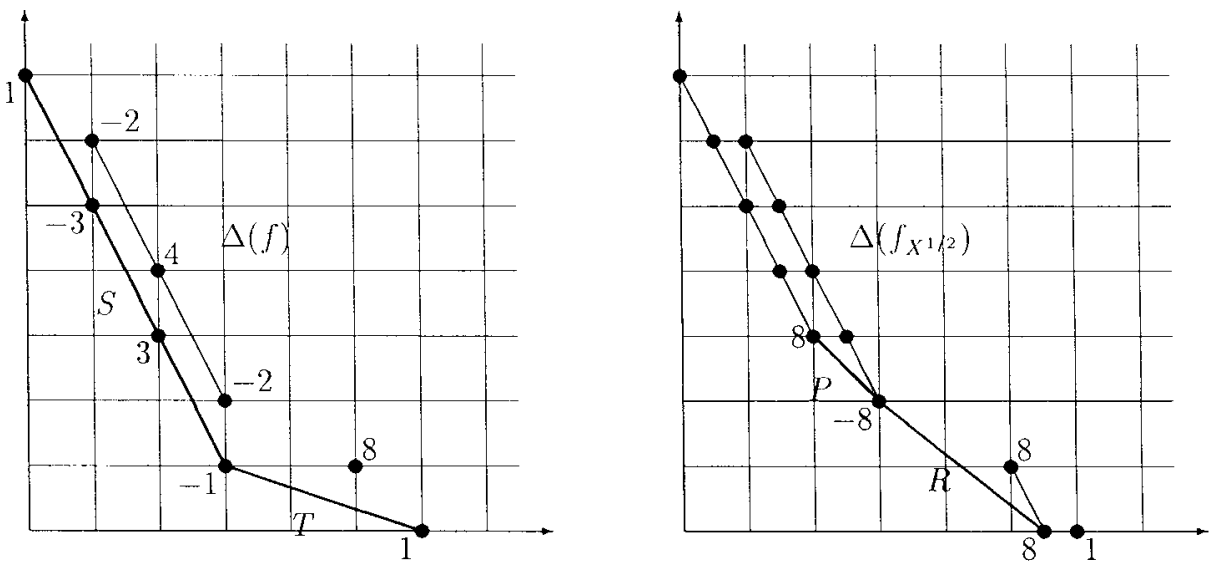

Hence $\overline{\mathscr{Q}}(f, X)=\mathscr{A}_{S} \oplus \mathscr{B}_{S}=\langle 7 / 2,7 / 2\rangle \oplus \mathscr{B}_{S}$, where $\mathscr{B}_{S}$ contains four quotients strictly greater then $7 / 2$. Consider two multiple nonzero roots $X^{1 / 2}$ and $-X^{1 / 2}$ of $\operatorname{in}(f, S)$. We have $\mathscr{B}_{S}=\overline{\mathscr{Q}}_{X^{1 / 2}}(f, X) \oplus \overline{\mathscr{Q}}_{-X^{1 / 2}}(f, X)$. Taking $X^{1 / 2}$ as a track we obtain $f_{X^{1 / 2}}=f\left(X, X^{1 / 2}+Y\right)=\left(Y+X^{1 / 2}\right) Y^{3}\left(Y+2 X^{1 / 2}\right)^{3}-$ $2 X\left(Y+X^{1 / 2}\right)^{2} Y^{2}\left(Y+2 X^{1 / 2}\right)^{2}+8 X^{5}\left(Y+X^{1 / 2}\right)+X^{6}$. The polygon $\mathscr{N}_{X^{1 / 2}}$ has two faces: $P$ which joins $(2,3)$ with $(3,2)$ and $R$ which joins $(3,2)$ with 
$(11 / 2,0)$. We have $\overline{\mathscr{Q}}_{X^{1 / 2}}(f, X)=\mathscr{A}_{X^{1 / 2}, P} \oplus \mathscr{B}_{X^{1 / 2}, P} \oplus \mathscr{A}_{X^{1 / 2}, R} \oplus \mathscr{B}_{X^{1 / 2}, R} \oplus$ $\mathscr{C}_{X^{1 / 2}}$. Note that in $\left(f_{X^{1 / 2}}, P\right)=8 X^{2} Y^{3}-8 X^{3} Y^{2}=8 X^{2} Y^{2}(Y-X), \alpha(P)=5$, $t\left(f_{X^{1 / 2}}, P\right)=2, \quad d\left(f_{X^{1 / 2}}, P\right)=0 ; \quad \operatorname{in}\left(f_{X^{1 / 2}}, R\right)=-8 X^{3} Y^{2}+8 X^{11 / 2}=-8 X^{3}$. $\left(Y-X^{5 / 4}\right)\left(Y+X^{5 / 4}\right), \quad \alpha(R)=11 / 2, \quad t\left(f_{X^{1 / 2}}, R\right)=2, \quad d\left(f_{X^{1 / 2}}, R\right)=0 \quad$ and $\delta\left(f_{X^{1 / 2}}\right)=0$. Since $\mathscr{B}_{X^{1 / 2}, P}=\mathscr{B}_{X^{1 / 2}, R}=\mathscr{C}_{X^{1 / 2}}=\langle\rangle$ we finish with $\overline{\mathscr{Q}}_{X^{1 / 2}}(f, X)=$ $\mathscr{A}_{X^{1 / 2}, P} \oplus \mathscr{A}_{X^{1 / 2}, R}=\langle 5,11 / 2\rangle$. Analogously, $\quad \overline{\mathscr{Q}}_{-X^{1 / 2}}(f, X)=\langle 5,11 / 2\rangle$ and finally $\overline{\mathscr{Q}}(f, X)=\mathscr{A}_{0}(f, X) \oplus \overline{\mathscr{Q}}_{X^{1 / 2}}(f, X) \oplus \overline{\mathscr{Q}}_{-X^{1 / 2}}(f, X)=\langle 7 / 2,7 / 2,5,5,11 / 2$, $11 / 2\rangle$. As a result we see that $f$ is reduced. We can compute the Milnor number from Teissier's formula $\mu_{0}(f)=\sum \mathscr{2}(f, X)-$ ord $f(0, Y)+1=22$. Note that $T_{\min }(f, X)=\left\{0, X^{1 / 2},-X^{1 / 2}\right\}$.

\section{Proof of the main result}

The proof of the main result will be performed in several steps. The first step is the classical Newton-Puiseux theorem, which provides a description of the Puiseux roots of a series in terms of its Newton polygon (Theorem 3.1). The second and fundamental step is the description of the Newton polygon of the derivative. In the final steps of the proof we applicate these methods to the Newton algorithm.

\section{The Newton-Puiseux theorem}

Let $f \in \mathbf{k}\left[\left[X^{*}, Y\right]\right]$ be a series such that $p=\operatorname{ord} f(0, Y)>1$. Let Zer $f=$ $\left\langle y_{1}(X), \ldots, y_{p}(X)\right\rangle$ be the system of all the positive-order-roots of $f=0$ in $\mathbf{k}[[X]]^{*}$. Consider $S \in \mathscr{N}(f)$ and define the form $\operatorname{in}(f, S)^{\circ}$ by the equation $\operatorname{in}(f, S)=X^{a_{S}} Y^{b_{S}} \operatorname{in}(f, S)^{\circ}$ where $a_{S}$ and $b_{S}$ are the maximal possible powers. Let us note that $\operatorname{deg} \operatorname{Zer} \operatorname{in}(f, S)^{\circ}=|S|_{2}$. For any $\theta>0$ (or $\theta=-\infty$ ) we define $\operatorname{Zer}^{\theta} f$, which contains those roots of Zer $f$ which satisfy ord $y_{i}(X)>\theta$. By the height of $\mathscr{N}^{\theta}(f)$ we mean $\sum_{S \in \mathcal{N}^{\theta}(f)}|S|_{2}$ and we denote it by $\left|\mathscr{N}^{\theta}(f)\right|$. We need the Newton-Puiseux theorem in the following form (we use convention in $0=0)$.

TheOREM 3.1. Let $\operatorname{Zer}^{\theta} f=\left\langle y_{1}(X), \ldots, y_{s}(X)\right\rangle$. Then

(i) $\left\langle\right.$ ord $y_{1}(X), \ldots$, ord $\left.y_{s}(X)\right\rangle=\oplus_{S \in \mathscr{N}^{\theta}(f)}\left\langle|S|_{1} /|S|_{2}:|S|_{2}\right\rangle \oplus$ $\langle+\infty: \delta(f)\rangle$

(ii) $\left\langle\right.$ in $y_{1}(X), \ldots$, in $\left.y_{s}(X)\right\rangle=\bigoplus_{S \in \mathscr{N}^{\theta}(f)} \operatorname{Zerin}(f, S)^{\circ} \oplus\langle 0: \delta(f)\rangle$.

(iii) $s=\left|\mathscr{N}^{\theta}(f)\right|+\delta(f)$.

The following property geometrically expresses the orders from (1). For $\theta>0$ and any closed nonempty subset $Z \subset \mathbf{R}_{+}^{2}$ we define the number

$$
\alpha(\theta, Z)=\min \{\alpha+\beta \theta:(\alpha, \beta) \in Z\} .
$$

Obviously $\alpha\left(\theta, \Delta_{f}\right)=\alpha(\theta$, supp $f)$. This number is the abscissa of the point where the line of inclination $\theta$, supporting $\Delta(f)$, intersects the horizontal axis. We have the following simple 
Property 3.2. Let $z(X) \in \mathbf{k}[[X]]^{*}, 0<$ ord $z(X)<+\infty$. Then

(a) ord $f(X, z(X)) \geq \alpha\left(\right.$ ord $\left.z, \Delta_{f}\right)$,

(b) the inequality is sharp if and only if there exists a face $S \in \mathscr{N}(f)$ such that $|S|_{1} /|S|_{2}=\operatorname{ord} z(X)$ and in $z(X)$ is a root of in $(f, S)$.

\section{Polygon of the derivative}

In this next step we describe the polygon $\mathscr{N}(\partial f / \partial Y)$. By eliminating the points from supp $f$ that lie on the horizontal axis and by moving all remaining points one unit down, we obtain the support of $\partial f / \partial Y$. In effect, if $S \in \mathscr{N}(f)$ does not touch the horizontal axis, then $T=S-(0,1)$ is a face of the polygon $\mathscr{N}(\partial f / \partial Y)$ and

$$
\text { in }\left(\frac{\partial f}{\partial Y}, T\right)=\frac{\partial}{\partial Y} \operatorname{in}(f, S) .
$$

If $S$ touches the horizontal axis then there exists the corresponding family of faces $T \in \mathscr{N}(\partial f / \partial Y)$ such that $|T|_{1} /|T|_{2} \geq|S|_{1} /|S|_{2}$ and $\sum|T|_{2}+\delta(\partial f / \partial Y)=$ $|S|_{2}-1$. If there exists an element $T$, of the family which is parallel to $S$, then (2) is also satisfied. As a result we have

Corollary 3.3 (see [L1], Corollary 5.4). Let $T \in \mathscr{N}(\partial f / \partial Y)$.

(a) If $T$ is parallel to face $S \in \mathcal{N}(f)$, then (2) is satisfied.

(b) If $T$ is not parallel to any face of $\mathscr{N}(f)$, then

- $|T|_{1} /|T|_{2}>|S|_{1} /|S|_{2}$ for every $S \in \mathscr{N}(f)$,

.. the polygon $\mathscr{N}(f)$ touches the horizontal axis.

We need a more detailed analysis of the relations between roots of in $(f, S)$ and $\partial / \partial Y \operatorname{in}(f, S)$. Consider the factorization

$$
\operatorname{in}(f, S)=b X^{a_{S}} Y^{b_{S}} L_{1}^{r_{1}} \cdots L_{k}^{r_{k}},
$$

where $Y, L_{1}, \ldots, L_{k}$ are different monic linear factors of $\operatorname{in}(f, S)$ in $\mathbf{k}[X]^{*}[Y]$ $\left(b \neq 0, k>0, r_{i}>0\right)$. Every factor has the form $Y-a X^{\theta}$, where $\theta=|S|_{1} /|S|_{2}$. We have the following

LEMMA 3.4 (see [LMP], Lemma 4.1).

(i) If $S$ does not touch the horizonatal axis $\left(b_{S}>0\right)$, then

$$
\frac{\partial}{\partial Y} \operatorname{in}(f, S)=b^{\prime} X^{a_{S}} Y^{b_{S}-1} L_{1}^{r_{1}-1} \cdots L_{k}^{r_{k}-1} L_{1}^{\prime} \cdots L_{k}^{\prime},
$$

where $L_{1}^{\prime}, \ldots, L_{k}^{\prime}$ are monic linear factors different from $Y, L_{1}, \ldots, L_{k}$.

(ii) If $S$ touches the horizontal axis $\left(b_{S}=0\right)$ and $k>1$ then

$$
\frac{\partial}{\partial Y} \operatorname{in}(f, S)=b^{\prime} X^{a_{S}} L_{1}^{r_{1}-1} \cdots L_{k}^{r_{k}-1} L_{1}^{\prime} \cdots L_{k-1}^{\prime},
$$

where $L_{1}^{\prime}, \ldots, L_{k-1}^{\prime}$ are monic linear factors different from $L_{1}, \ldots, L_{k}$. 
Proof. If $b_{S}>0$ then $Y$-differentiation of $\operatorname{in}(f, S)=X^{a_{S}} Y^{b_{S}} \operatorname{in}(f, S)^{\circ}$ moves the support of $\operatorname{in}(f, S)$ by the vector $(0,-1)$. We obtain

$$
\frac{\partial}{\partial Y} \operatorname{in}(f, S)=X^{a_{S}} Y^{b_{S}-1} h(X, Y),
$$

where the powers $a_{S}$ and $b_{S}-1$ are the maximal possible. Let us consider a factor of $\operatorname{in}(f, S)$ of the form $L^{r}=\left(Y-a X^{\theta}\right)^{r}$. By substitution $a X^{\theta}+Y$ in place of $Y$, we obtain $Y^{r}$ in place of $L^{r}$. We use (4) to show that $L^{r-1}$ divides $\partial / \partial Y$ in $(f, S)$ and $r-1$ is the maximal possible power.

Remark 3.5. If $S$ touches the horizontal axis $\left(b_{S}=0\right)$ and $k=1$ in (3), then $\operatorname{in}(f, S)=b X^{a_{S}} L^{r}$ and $\partial / \partial Y(\operatorname{in}(f, S))=b^{\prime} X^{a_{S}} L^{r-1}$.

From the above lemma and the previous statements we have

Proposition 3.6. Let $S \in \mathscr{N}(f)$.

(a) If $t=t(f, S)>1$ then there exists $t-1$ solutions $\psi_{1}^{\prime}(X), \ldots, \psi_{t-1}^{\prime}(X)$ of $\partial f / \partial Y=0$ such that ord $f\left(X, \psi_{i}^{\prime}(X)\right)=\alpha(S)$.

(b) If $d=d(f, S)>0$ then there exists $d$ solutions $\psi_{1}(X), \ldots, \psi_{d}(X)$ of $\partial f / \partial Y=0$ such that ord $f\left(X, \psi_{i}(X)\right)>\alpha(S)$.

Proof. (a) If $S$ does not touch the horizontal axis, then by using notations of Lemma 3.4, $k=t(f, S)-1$. Since $T=S-(0,1)$ is a face of $\mathscr{N}(\partial f / \partial Y)$, from Theorem 3.1 there exist solutions $\psi_{1}^{\prime}(X), \ldots, \psi_{k}^{\prime}(X)$ of $\partial f / \partial Y=0$ that correspond to the factor $L_{1}^{\prime} \cdots L_{k}^{\prime}$ of $\partial / \partial Y(\operatorname{in}(f, S))=\operatorname{in}(\partial f / \partial Y, T)$. Clearly ord $\psi_{i}^{\prime}(X)=|T|_{1} /|T|_{2}=|S|_{1} /|S|_{2}$. Since $L_{1}^{\prime}, \ldots, L_{k}^{\prime}$ are different from $Y, L_{1}, \ldots$, $L_{k}$, then in $\psi_{i}^{\prime}$ is not a root of $\operatorname{in}(f, S)$. By Property 3.2 we obtain

$$
\text { ord } f\left(X, \psi_{i}^{\prime}(X)\right)=\alpha\left(|S|_{1} /|S|_{2}, \Delta_{f}\right)=\alpha(S) \text {. }
$$

If $S$ touches the horizontal axis, then $k=t(f, S)$. Let $s$ be the number of appearances of $Y$ in the sequence $L_{1}^{\prime}, \ldots, L_{k-1}^{\prime}$. We can assume that $L_{1}^{\prime}, \ldots$, $L_{k-s-1}^{\prime}$ are different from $Y$. Analogously, as before, we construct solutions $\psi_{1}^{\prime}(X), \ldots, \psi_{k-s-1}^{\prime}(X)$ that satisfy (5). For $s>0$, by the Newton-Puiseux theorem, there exist $s$ solutions $\psi_{k-s}^{\prime}(X), \ldots, \psi_{k-1}^{\prime}(X)$ of $\partial f / \partial Y=0$ that correspond to the family of faces $T \in \mathscr{N}(\partial f / \partial Y)$, that lie below the line $\beta=s$, and to the distance $\delta(\partial f / \partial Y)$. We have $\sum|T|_{2}+\delta(\partial f / \partial Y)=s$. If $\psi_{i}^{\prime}(X)$ comes from a face $T$ of the family, then by Corollary 3.3 we have ord $\psi_{1}(X)=|T|_{1} /|T|_{2}>$ $|S|_{1} /|S|_{2}$. Since $S$ touches the horizontal axis, it is the lower possible face of $\mathscr{N}(f)$. Therefore, the line supporting $\Delta_{f}$, which is parallel to $T$, meets $\Delta_{f}$ at the vertex lying on the horizontal axis. The vertex has the abscissa ord $f(X, 0)$. From Property 3.2 we obtain ord $f\left(X, \psi_{i}^{\prime}(X)\right)=\alpha\left(|T|_{1} /|T|_{2}, \Delta_{f}\right)=$ ord $f(X, 0)$. If $\psi_{i}(X)=0$, then similarly ord $f\left(X, \psi_{i}^{\prime}(X)\right)=$ ord $f(X, 0)$. Since ord $f(X, 0)=$ $\alpha(S)$ we finish with (5) in both cases.

(b) Note that $d=d(f, S)=\left(r_{1}-1\right)+\cdots+\left(r_{k}-1\right)$. If $d>0$ then there exists a face $T \in \mathscr{N}(\partial f / \partial Y)$ which is parallel to $S$. By Theorem 3.1 and by 
Lemma 3.4 there exist solutions $\psi_{1}(X), \ldots, \psi_{d}(X)$ of $\partial f / \partial Y=0$ corresponding to the factor $L_{1}^{r_{1}-1} \cdots L_{k}^{r_{k}-1}$ of $\partial / \partial Y(\operatorname{in}(f, S))=\operatorname{in}(\partial f / \partial Y, T)$. Clearly ord $\psi_{i}(X)=$ $|T|_{1} /|T|_{2}=|S|_{1} /|S|_{2}$ and in $\psi_{i}(X)$ is a root of $\operatorname{in}(f, S)$. From Property 3.2 it follows that ord $f\left(X, \psi_{i}(X)\right)>\alpha\left(|S|_{1} /|S|_{2}, \Delta_{f}\right)=\alpha(S)$, which completes the proof.

\section{The Newton polygon relative to a Puiseux polynomial}

Let $\varphi \in \mathbf{k}[X]^{*}$ be an arbitrary Puiseux polynomial of a positive order. We begin by describing the polygon $\mathscr{N}_{\varphi}=\mathscr{N}^{\operatorname{deg} \varphi}\left(f_{\varphi}\right)$. Applying the Weierstrass preparation theorem we can write

$$
f(X, Y)=U(X, Y)\left(Y-y_{1}(X)\right) \cdots\left(Y-y_{p}(X)\right),
$$

where $U(X, Y) \in \mathbf{k}[[X, Y]]$ is a unit and, as before, Zer $f=\left\langle y_{1}, \ldots, y_{p}\right\rangle$. Hence

$$
f_{\varphi}(X, Y)=f(X, \varphi(X)+Y)=U^{\prime}(X, Y)\left[Y-\left(y_{1}-\varphi\right)\right] \cdots\left[Y-\left(y_{p}-\varphi\right)\right],
$$

where $U^{\prime}(X, Y) \in \mathbf{k}\left[\left[X^{*}, Y\right]\right]$ is also a unit. Therefore

$$
\text { Zer } f_{\varphi}=\left\langle y_{1}-\varphi, \ldots, y_{p}-\varphi\right\rangle \text {. }
$$

Using Theorem 3.1 (a) with $\theta=-\infty$ we obtain

$$
\left\langle\operatorname{ord}\left(y_{1}-\varphi\right), \ldots, \operatorname{ord}\left(y_{p}-\varphi\right)\right\rangle=\bigoplus_{S \in \mathcal{N}\left(f_{\varphi}\right)}\left\langle|S|_{1} /|S|_{2}:|S|_{2}\right\rangle \oplus\left\langle+\infty: \delta\left(f_{\varphi}\right)\right\rangle .
$$

Let $\operatorname{Zer}_{\varphi} f=\left\langle y_{1}, \ldots, y_{s}\right\rangle$ denote the system of such solutions from Zer $f$ that $\operatorname{ord}\left(y_{i}-\varphi\right)>\operatorname{deg} \varphi$ (i.e. $y_{1}(X), \ldots, y_{s}(X)$ are continuations of $\varphi$ ). As a consequence of Theorem 3.1 applied with $\theta=\operatorname{deg} \varphi$ we have

COROLlary 3.7.

(a) $\left\langle\operatorname{ord}\left(y_{1}-\varphi\right), \ldots, \operatorname{ord}\left(y_{s}-\varphi\right)\right\rangle=\bigoplus_{S \in \mathcal{N}_{\varphi}}\left\langle|S|_{1} /|S|_{2}:|S|_{2}\right\rangle \oplus\left\langle+\infty: \delta\left(f_{\varphi}\right)\right\rangle$

(b) $\left\langle\operatorname{in}\left(y_{1}-\varphi\right), \ldots, \operatorname{in}\left(y_{p}-\varphi\right)\right\rangle=\oplus_{S \in \mathcal{N}_{\varphi}} \operatorname{Zer} \operatorname{in}\left(f_{\varphi}, S\right)^{\circ} \oplus\left\langle 0: \delta\left(f_{\varphi}\right)\right\rangle$

(c) $s=\left|\mathscr{N}_{\varphi}\right|+\delta\left(f_{\varphi}\right)$.

We will need the following simple

Property 3.8. Let $g \in \mathbf{k}\left[\left[X^{*}, Y\right]\right]$ be a nonzero series. Fix $\theta>0$.

(i) There exists a unique representation

$$
g=g_{0}+g_{1}+g_{2}+\cdots
$$

such that every $g_{i}$ is a quasi-homogeneous form of the weights $(\theta, 1)$.

(ii) If $r$ is the maximal power such that $Y^{r}$ divides $g_{0}$, then

$$
\left|\mathscr{N}^{\theta}(g)\right|+\delta(g)=r .
$$

(iii) If there exists $S \in \mathscr{N}(g)$ such that $|S|_{1} /|S|_{2}=\theta$, then $g_{0}=\operatorname{in}(f, S)$.

Now, we can prove the following 
Proposition 3.9. Assume that $\varphi \in \mathbf{k}[X]^{*}$ is such a polynomial that $\mathscr{N}_{\varphi}$ is nonempty. Let $S \in \mathscr{N}_{\varphi}$ and let $a X^{\theta}$ be a nonzero root of $\operatorname{in}(f, S)$. Then

$$
\left|\mathscr{N}_{\varphi+a X^{\theta}}\right|+\delta\left(f_{\varphi+a X^{\theta}}\right)=\text { mutiplicity of a } X^{\theta} \text { as a root of } \operatorname{in}(f, S) .
$$

Proof. For $\theta=|S|_{1} /|S|_{2}$ let us consider the representation

$$
f_{\varphi}=g_{0}+g_{1}+g_{2}+\cdots
$$

according to Property 3.8. We have $g_{0}=\operatorname{in}\left(f_{\varphi}, S\right)$. Let us write $\operatorname{in}\left(f_{\varphi}, S\right)=$ $\left(Y-a X^{\theta}\right)^{r} h(X, Y)$, where $r$ is the maximal possible power. We have $r>0$. Let us note that $f_{\varphi+a X^{\theta}}(X, Y)=f_{\varphi}\left(X, a X^{\theta}+Y\right)$. Hence

$$
f_{\varphi+a X^{\theta}}=\tilde{g}_{0}+\tilde{g}_{1}+\tilde{g}_{2}+\cdots,
$$

where $\tilde{g}_{i}(X, Y)=g_{i}\left(X, a X^{\theta}+Y\right)$. Since $a X^{\theta}+Y$ is the homogeneous form of the weights $(\theta, 1),(7)$ is the unique representation guaranteed by Property 3.8. We have $\tilde{g}_{0}(X, Y)=\operatorname{in}\left(f_{\varphi}, S\right)\left(X, a X^{\theta}+Y\right)=Y^{r} \tilde{h}(X, Y)$. From Property 3.8(b) follows that

$$
r=\left|\mathscr{N}_{\varphi+a X^{\theta}}\right|+\delta\left(f_{\varphi+a X^{\theta}}\right),
$$

which concludes the proof.

\section{Tracks of the Newton algorithm}

We give here two different characterizations of the set $T(f, X)$, of the tracks of the Newton algorithm for $f$, and prove their equivalency with the definition from Section 2. Recall

Definition 3.10. $T(f, X) \subset \mathbf{k}[X]^{*}$ is the minimal subset satisfying two properties: (I) $0 \in T(f, X)$, (II) for every $\varphi(X) \in T(f, X)$, if there exists $S \in \mathscr{N}_{\varphi}$, then for every nonzero root $a X^{\theta}$ of $\operatorname{in}\left(f_{\varphi}, S\right), \varphi+a X^{\theta} \in T(f, X)$.

Let us define

$$
T_{1}(f, X)=\left\{\varphi \in \mathbf{k}[X]^{*}: \exists y(X) \in \operatorname{Zer} f \text { such that } \operatorname{ord}(y(X)-\varphi)>\operatorname{deg} \varphi\right\}
$$

and

$$
T_{2}(f, X)=\left\{\varphi \in \mathbf{k}[X]^{*}:\left|\mathscr{N}_{\varphi}\right|+\delta\left(f_{\varphi}\right)>0\right\} .
$$

Proposition 3.11. If Zer $f$ is nonempty then $T(f, X)=T_{1}(f, X)=$ $T_{2}(f, X)$.

Proof. The equality $T_{1}(f, X)=T_{2}(f, X)$ follows directly from Corollary 3.7 (c). Since $T(f, X)$ is the minimal set with properties (I) and (II), it suffices to show that $T_{2}(f, X)$ satisfies both properties in order to verify that $T(f, X) \subset$ $T_{2}(f, X)$. Because Zer $f$ is nonempty, the first condition $0 \in T_{2}(f, X)$ is clear. In order to check the second condition, let us consider $\varphi \in T_{2}(f, X)$. Let us assume that there exists $S \in \mathscr{N}_{\varphi}$ and let $a X^{\theta}$ be an arbitrary nonzero root of 
$\operatorname{in}\left(f_{\varphi}, S\right)$. We must show that $\varphi+a X^{\theta} \in T_{2}(f, X)$, which follows immediately from Proposition 3.9.

To finish the proof it suffices to verify that $T_{1}(f, X) \subset T(f, X)$. Let $\varphi=a_{1} X^{\theta_{1}}+\cdots+a_{n} X^{\theta_{n}}$ be a non zero element of $T_{1}(f, X)$ i.e. there exists $y(X) \in \operatorname{Zer} f$ such that $\operatorname{ord}(y(X)-\varphi)>\operatorname{deg} \varphi$. Let us put $\varphi_{0}=0$ and $\varphi_{k}=$ $a_{1} X^{\theta_{1}}+\cdots+a_{k} X^{\theta_{k}}$ for $k=1, \ldots, n-1$. We will show the implication $\varphi_{k} \in$ $T(f, X) \Rightarrow \varphi_{k+1} \in T(f, X)$. Assume that $\varphi_{k} \in T(f, X)$. We have $\operatorname{in}\left(y(X)-\varphi_{k}\right)=$ $a_{k+1} X^{\theta_{k+1}}$. By Corollary 3.7 there exists $S \in \mathscr{N}_{\varphi_{k}}$ such that $a_{k+1} X^{\theta_{k+1}}$ is a root of $\operatorname{in}\left(f_{\varphi_{k}}, S\right)$. From property (II) we obtain $\varphi_{k+1}=\varphi_{k}+a_{k+1} X^{\theta_{k+1}} \in T(f, X)$. Since $0 \in T(f, X)$, by induction we show that $\varphi=\varphi_{n} \in T(f, X)$, completing the proof.

\section{End of the proof}

We are in a good position to finish the proof of the main result (Theorem 2.1).

Proof of (a). Let $\varphi \in T(f, X)$. By Proposition $3.11 s=\left|\mathscr{N}_{\varphi}\right|+\delta\left(f_{\varphi}\right)>0$. By Theorem 3.1 there exist solutions $\psi_{1}(X), \ldots, \psi_{s}(X)$ of $f_{\varphi}=0$ such that $\operatorname{deg} \psi_{i}>\operatorname{deg} \varphi$. By using previous notation we can write $\operatorname{Zer}^{\operatorname{deg} \varphi}\left(f_{\varphi}\right)=$ $\left\langle\psi_{1}, \ldots, \psi_{s}\right\rangle$. On the other hand, we have the system $\operatorname{Zer}_{\varphi} f=\left\langle y_{1}, \ldots, y_{s}\right\rangle$ of roots of $f=0$ which are continuations of $\varphi$. Corollary 3.7 states the one-to-one correspondence between $\operatorname{Zer}^{\operatorname{deg} \varphi}\left(f_{\varphi}\right)$ and $\operatorname{Zer}_{\varphi} f$ by $\psi \mapsto y=\varphi+\psi$ (the inverse: $y \mapsto \psi=y-\varphi)$. Since $(\partial f / \partial Y)_{\varphi}=\partial f_{\varphi} / \partial Y$, this construction can be directly applied to $\operatorname{Zer}_{\varphi}(\partial f / \partial Y)$. If $s=\left|\mathscr{N}_{\varphi}\right|+\delta\left(f_{\varphi}\right)>1$, then by $Y$-differentiation and by Theorem 3.1, there exist $s-1$ solutions $\psi_{1}, \ldots, \psi_{s-1}$ of $\partial f_{\varphi} / \partial Y=0$ such that ord $\psi_{i}>\operatorname{deg} \varphi$. Clearly

$$
\operatorname{Zer}_{\varphi}(\partial f / \partial Y)=\left\langle\phi+\psi_{1}, \ldots, \phi+\psi_{s-1}\right\rangle .
$$

Consider $S \in \mathscr{N}_{\varphi}$. If $t=t\left(f_{\varphi}, S\right)>1$, then by Proposition 3.6 (a) there exist $t-1$ solutions $\psi_{1}^{\prime}, \ldots, \psi_{t-1}^{\prime}$ of $\partial f_{\varphi} / \partial Y=0$ such that ord $f_{\varphi}\left(X, \psi_{i}^{\prime}(X)\right)=\alpha(S)$. Then for $z_{i}^{\prime}(X)=\varphi(X)+\psi_{i}^{\prime}(X) \in \operatorname{Zer}_{\varphi}(\partial f / \partial Y)$ we have

ord $f\left(X, z_{i}^{\prime}(X)\right)=$ ord $f\left(X, \varphi(X)+\psi_{i}^{\prime}(X)\right)=\operatorname{ord} f_{\varphi}\left(X, \psi_{i}^{\prime}(X)\right)=\alpha(S)$.

If $d=d(f, S)>0$ then by Proposition 3.6 (b) we analogously construct solutions $z_{1}(X), \ldots, z_{d}(X) \in \operatorname{Zer}_{\varphi}(\partial f / \partial Y)$ such that ord $f\left(X, z_{i}(X)\right)>\alpha(S)$. We finish with the observation that if $\delta\left(f_{\varphi}\right)>1$, then $\varphi(X)$ is a common root of $f=0$ and $\partial f / \partial Y=0$ with multiplicity $\delta\left(f_{\varphi}\right)-1$ and ord $f(X, \varphi(X))=$ ord $f_{\varphi}(X, 0)=+\infty$.

Proof of (b). Consider roots $z_{1}(X), \ldots, z_{d}(X) \in \operatorname{Zer}_{\varphi}(\partial f / \partial Y)$ such that

$$
\mathscr{B}_{\varphi, S}=\left\langle\text { ord } f\left(X, z_{1}(X)\right), \ldots, \text { ord } f\left(X, z_{d}(X)\right)\right\rangle .
$$

Fix $i \in\{1, \ldots, d\}$. We must show the fact that $z_{i}$ has the form $\varphi+a X^{\theta}+\cdots$, where $a X^{\theta}$ is a multiple root of $\operatorname{in}\left(f_{\varphi}, S\right)$. Let $\psi_{i}=z_{i}-\varphi$. Since in $\psi_{i}=a X^{\theta}$, this fact follows immediately from the construction described in Proposition 3.6. 
Proof of (c). We begin with an observation that $T_{\min }(f, X)$ is finite (Remark 2.4). It is enough to consider the tracks generated by solutions of $f=0$ with infinite number of termes. Let us fix such a solution $y(X)=$ $a_{1} X^{\theta_{1}}+a_{2} X^{\theta_{2}}+\cdots \in \operatorname{Zer} f$. Let us define the sequence of tracks $\varphi_{0}=0, \ldots$, $\varphi_{j}=a_{1} X^{\theta_{1}}+\cdots+a_{j} X^{\theta_{j}}, \ldots$ and the sequences of series $f^{(j)}:=f_{\varphi_{j-1}}$ and polygons $\mathscr{N}^{(j)}:=\mathscr{N}_{\varphi_{j-1}}$. For any $j=1,2, \ldots$, according to Corollary 3.7 , there exists a face $S^{(j)} \in \mathscr{N}^{(j)}$ with the inclination $\theta_{j}$, such that $a_{j} X^{\theta_{j}}$ is a root of $\operatorname{in}\left(f^{(j)}, S^{(j)}\right)$ with multiplicity $r_{j}>0$. We write $l_{j}=\operatorname{deg}_{Y} \operatorname{in}\left(f^{(j)}, S^{(j)}\right)$. Of course, $l_{j} \geq r_{j}$. From Proposition 3.9 we have $r_{j}=\left|\mathscr{N}^{(j+1)}\right|+\delta\left(f^{(j+1)}\right) \geq l_{j+1}$. As the result we have the infinite sequence $l_{1} \geq r_{1} \geq l_{2} \geq r_{2} \geq \cdots$ of positive integers. Therefore, there exists $j$ such that $r_{j}=l_{j+1}=r_{j+1}=\cdots$. The first equality means that $S^{(j+1)}$ is the highest face of $\mathscr{N}^{(j)}$. The second equality means that $\operatorname{in}\left(f^{(j+1)}, S^{(j+1)}\right)$ has a unique nonzero root, hence $t\left(f^{(j+1)}, S^{(j+1)}\right)=1$. Since $S^{(j+1)}$ touches the horizontal axis (Remark 3.5), $\mathscr{N}^{(j+1)}$ has only one face.

To conclude the proof we need to show that for every solution $z(X) \in$ $\operatorname{Zer}(\partial f / \partial Y)$, such that

$$
\text { ord } f(X, z(X))<+\infty
$$

there exists a track $\varphi \in T(f, X)$ and a face $S \in \mathscr{N}_{\varphi}$ such that ord $f(X, z(X))=$ $\alpha(S)$. According to (6) and (9) we have $\operatorname{ord}(z(X)-y(X))<+\infty$ for every $y(X) \in \operatorname{Zer} f$. Let us choose a solution $y(X)$ with the longest common track $\varphi(X)$ of both series $z(X)$ and $y(X)$. We have $z(X)=\varphi(X)+\psi_{z}(X)$ and $y(X)=$ $\varphi(X)+\psi_{y}(X)$, where ord $\psi_{z}>\operatorname{deg} \varphi, \operatorname{ord} \psi_{y}>\operatorname{deg} \varphi$ and in $\psi_{y} \neq$ in $\psi_{z}$. Since $0=(\partial f / \partial Y)(X, z(X))=\left(\partial f_{\varphi} / \partial Y\right)\left(X, \psi_{z}(X)\right)$, therefore in $\psi_{z}$ can be described by $\mathscr{N}_{\varphi}^{\prime}:=\mathscr{N}^{\operatorname{deg} \varphi}\left(\partial f_{\varphi} / \partial Y\right)$ by virtue of Corollary 3.7.

If ord $\psi_{z}=+\infty$, then $z(X)=\varphi(X)$ and according to (9) we have ord $f(X, z(X))=$ ord $f(X, \varphi(X))=$ ord $f_{\varphi}(X, 0)<+\infty$. Hence, there exists $S \in \mathscr{N}_{\varphi}$ which touches the horizontal axis and ord $f_{\varphi}(X, 0)=\alpha(S)$. If ord $\psi_{z}(X)$ is finite, but does not appear as an inclination of any face of $\mathscr{N}_{\varphi}$, then according Corollary 3.3 (b) the line supporting $\Delta\left(f_{\varphi}\right)$, of inclination ord $\psi_{z}$, intersects the horizontal axis at the point $\left(\right.$ ord $\left.f_{\varphi}(X, 0), 0\right)$. Hence, by Property 3.2 we have ord $f(X, z(X))=$ ord $f_{\varphi}\left(X, \psi_{z}(X)\right)=$ ord $f_{\varphi}(X, 0)$. Therefore, in both cases ord $f(X, z(X))=\alpha(S)$.

Let us assume that ord $\psi_{z}=|S|_{1} /|S|_{2}$ for a certain $S \in \mathscr{N}_{\varphi}$ but that in $\psi_{z}$ is not a root of $\operatorname{in}\left(f_{\varphi}, S\right)$. By Property 3.2 we have ord $f(X, z(X))=\alpha(S)$ as before. If in $\psi_{z}$ is a root of $\operatorname{in}\left(f_{\varphi}, S\right)$, then by Theorem 3.1 there exists $y(X) \in \operatorname{Zer} f$ of the form $y(X)=\varphi(X)+\psi_{z}(X)+\cdots$, which contradicts the definition of $y(X)$. Because of this contradiction, the last possibility cannot happen and we conclude the proof of the main result.

\section{Eggers' example}

The following example shows that curves which are not equisingular can have the same polar invariants, counting their multiplicities. Let us consider the following four Puiseux series 


$$
\begin{array}{ll}
y_{1}=X+X^{3 / 2}+X^{15 / 4}, & y_{3}=X+X^{5 / 2}+X^{11 / 4}, \\
y_{2}=2 X+X^{5 / 2}+X^{13 / 4}, & y_{4}=2 X+X^{3 / 2}+X^{17 / 4} .
\end{array}
$$

Let $f_{1}, f_{2}, f_{3}, f_{4} \in \mathbf{k}[[X, Y]]$ be the minimal series respectively for $y_{1}, y_{2}, y_{3}, y_{4}$. We put $f=f_{1} f_{2}$ and $f^{\prime}=f_{3} f_{4}$. Let us begin with $f$. We have the following cycle for $y_{1}$

as well as for $y_{2}$

$$
y_{1}^{(i)}=\varepsilon^{4 i} X^{4 / 4}+\varepsilon^{6 i} X^{6 / 4}+\varepsilon^{15 i} X^{15 / 4}
$$

$$
y_{2}^{(i)}=2 \varepsilon^{4 i} X^{4 / 4}+\varepsilon^{10 i} X^{10 / 4}+\varepsilon^{13 i} X^{13 / 4},
$$

where $i=0,1,2,3$ and where $\varepsilon$ is a primitive root of unity of degree four. On the basis of the table provided below we can analyze tracks $0, X, X+X^{3 / 2}$ and $X-X^{3 / 2}$

\begin{tabular}{|c|c|c|c|}
\hline$f(X, Y)$ & $f(X, X+Y)$ & $f\left(X, X+X^{3 / 2}+Y\right)$ & $f\left(X, X-X^{3 / 2}+Y\right)$ \\
\hline$y_{1}^{(0)}=X+X^{3 / 2}+X^{15 / 4}$ & $X^{3 / 2}$ & $X^{15 / 4}$ & $2 X^{3 / 2}$ \\
$y_{1}^{(1)}=X-X^{3 / 2}-\varepsilon X^{15 / 4}$ & $-X^{3 / 2}$ & $-2 X^{3 / 2}$ & $-\varepsilon X^{15 / 4}$ \\
$y_{1}^{(2)}=X+X^{3 / 2}-X^{15 / 4}$ & $X^{3 / 2}$ & $-X^{15 / 4}$ & $2 X^{3 / 2}$ \\
$y_{1}^{(3)}=X-X^{3 / 2}+\varepsilon X^{15 / 4}$ & $-X^{3 / 2}$ & $-2 X^{3 / 2}$ & $\varepsilon X^{15 / 4}$ \\
$y_{2}^{(0)}=2 X+X^{5 / 2}+X^{13 / 4}$ & $X$ & $X$ & $X$ \\
$y_{2}^{(1)}=2 X-X^{5 / 2}+\varepsilon X^{13 / 4}$ & $X$ & $X$ & $X$ \\
$y_{2}^{(2)}=2 X+X^{5 / 2}-X^{13 / 4}$ & $X$ & $X$ & $X$ \\
$y_{2}^{(3)}=2 X-X^{5 / 2}-\varepsilon X^{13 / 4}$ & $X$ & $X$ & $X$ \\
\hline
\end{tabular}

The first column presents all the roots of $f=0$; the second-the initial forms of solutions of $f(X, X+Y)=0$; the third $-f\left(X, X+X^{3 / 2}+Y\right)=0$ and the fourth $-f\left(X, X-X^{3 / 2}+Y\right)=0$. This information allows us to reconstruct the relative polygons as well as their initial forms.

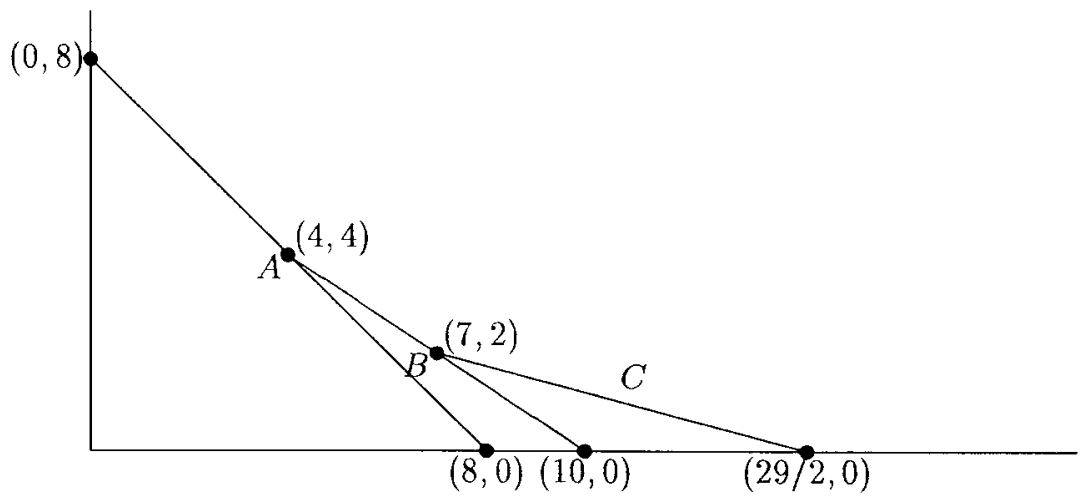


Polygon $\mathscr{N}(f)$ has one face $A$, which joins points $(0,8)$ and $(8,0), \operatorname{in}(f, A)=$ $(Y-X)^{4}(Y-2 X)^{4}$, thus $t=t(f, A)=2 ;$ from this we obtain invariant $\alpha(A)=8$ with multiplicity $t-1=1$. Polygon $\mathscr{N}_{X}$ has one face $B$, which joins points $(4,4)$ and $(10,0), \quad$ in $\left(f_{X}, B\right)=X^{4}\left(Y-X^{3 / 2}\right)^{2}\left(Y+X^{3 / 2}\right)^{2}$, thus $t=t\left(f_{X}, B\right)=2$; we obtain invariant $\alpha(B)=10$ with multiplicity $t-1=1$. Finally polygon $\mathscr{N}_{X+X^{3 / 2}}$ has a single face $C$, which joins $(7,2)$ and $(29 / 2,0), \quad \operatorname{in}\left(f_{X+X^{3 / 2}}, C\right)=4 X^{7}\left(Y-X^{15 / 4}\right)\left(Y+X^{15 / 4}\right)$, thus $t=t\left(f_{X+X^{3 / 2}}, C\right)$ $=2$; therefore we obtain invariant $\alpha(C)=29 / 2$ with multiplicity $t-1=1$. Taking into account track $X-X^{3 / 2}$ we again obtain $\alpha(C)=29 / 2$ with multiplicity 1 .

With the help of the second table we analyze tracks $2 X, 2 X+X^{5 / 2}$ as well as $2 X-X^{5 / 2}$.

\begin{tabular}{|c|c|c|c|}
\hline$f(X, Y)$ & $f(X, 2 X+Y)$ & $f\left(X, 2 X+X^{5 / 2}+Y\right)$ & $f\left(X, 2 X-X^{5 / 2}+Y\right)$ \\
\hline$y_{1}^{(0)}=X+X^{3 / 2}+X^{15 / 4}$ & $-X$ & $-X$ & $-X$ \\
$y_{1}^{(1)}=X-X^{3 / 2}-\varepsilon X^{15 / 4}$ & $-X$ & $-X$ & $-X$ \\
$y_{1}^{(2)}=X+X^{3 / 2}-X^{15 / 4}$ & $-X$ & $-X$ & $-X$ \\
$y_{1}^{(3)}=X-X^{3 / 2}+\varepsilon X^{15 / 4}$ & $-X$ & $-X$ & $-X$ \\
$y_{2}^{(0)}=2 X+X^{5 / 2}+X^{13 / 4}$ & $X^{5 / 2}$ & $X^{13 / 4}$ & $2 X^{5 / 2}$ \\
$y_{2}^{(1)}=2 X-X^{5 / 2}+\varepsilon X^{13 / 4}$ & $-X^{5 / 2}$ & $-2 X^{5 / 2}$ & $\varepsilon X^{13 / 4}$ \\
$y_{2}^{(2)}=2 X+X^{5 / 2}-X^{13 / 4}$ & $X^{5 / 2}$ & $-X^{13 / 4}$ & $2 X^{5 / 2}$ \\
$y_{2}^{(3)}=2 X-X^{5 / 2}-\varepsilon X^{13 / 4}$ & $-X^{5 / 2}$ & $-2 X^{5 / 2}$ & $-\varepsilon X^{13 / 4}$ \\
\hline
\end{tabular}

Polygon $\mathscr{N}_{2 X}$ has one segment $D$, which joins $(4,4)$ and $(14,0), \operatorname{in}\left(f_{2 X}, D\right)=$ $X^{4}\left(Y-X^{5 / 2}\right)^{2}\left(Y+X^{5 / 2}\right)^{2}$, thus $t=t\left(f_{2 X}, D\right)=2$; we obtain invariant $\alpha(D)=14$ with multiplicity $t-1=1$.

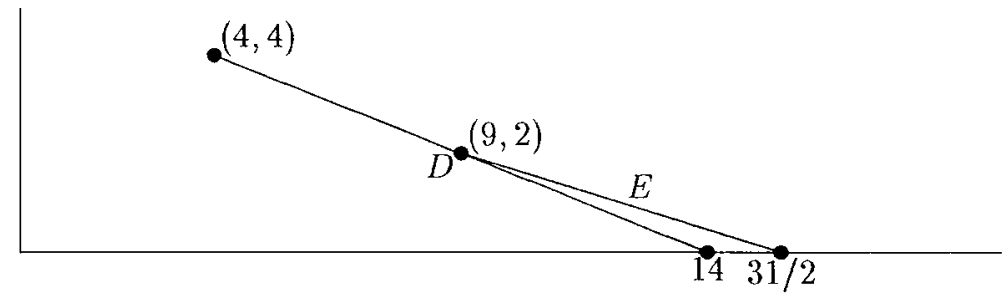

Polygon $\mathscr{N}_{2 X+X^{5 / 2}}$ contains one face $E$, which joins $(9,2)$ and $(31 / 2,0)$, $\operatorname{in}\left(f_{2 X+X^{5 / 2}}, E\right)=4 X^{9}\left(Y-X^{13 / 4}\right)\left(Y+X^{13 / 4}\right)$, thus $t=t\left(f_{2 X+X^{5 / 2}}, E\right)=2$; we obtain invariant $\alpha(E)=31 / 2$ with multiplicity $t-1=1$. Considering track $2 X-X^{5 / 2}$ we again obtain $\alpha(E)=31 / 2$ with multiplicity 1 . 
The remaining tracks do not lead to new solutions. The complete system of invariants is as follows

$$
\langle 8,10,14,29 / 2,29 / 2,31 / 2,31 / 2\rangle \text {. }
$$

An analogous analysis of series $f^{\prime}$ results in the same system. The idea behind Eggers' example becomes visible when we simultaneously mark faces $A, B, C$, $D, E$. Dotted lines signify new faces, which take the place of $C$ and $E$ for $f^{\prime}$.

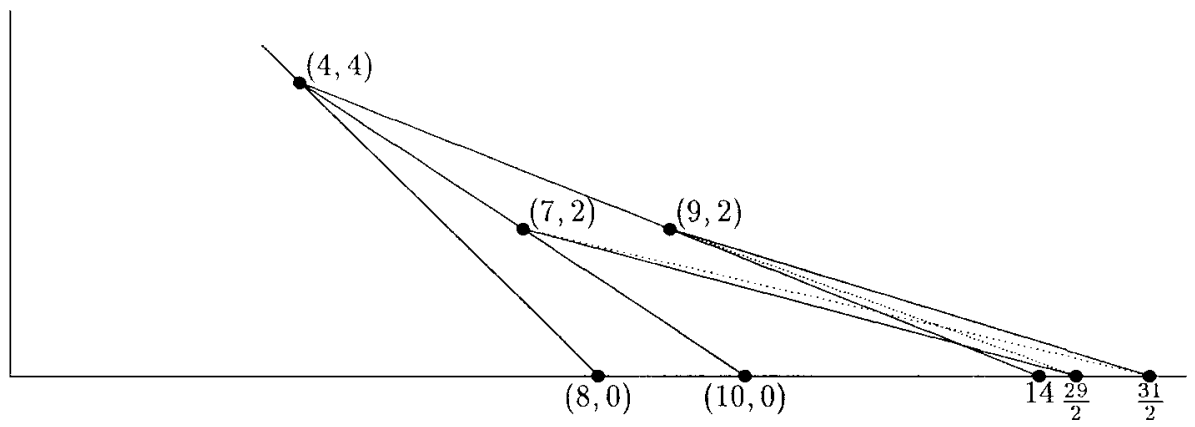

\section{The case of one branch}

Let us consider the case when $f \in \mathbf{k}[[X, Y]]$ is an irreducible series $Y$-regular of order $p(p>1)$. Let us fix Puiseux solution

$$
y(X)=a_{1} X^{v_{1} / p}+a_{2} X^{v_{2} / p}+\cdots
$$

of equation $f=0\left(a_{j} \neq 0,0<v_{1}<v_{2}<\right.$ integers, $\left.\operatorname{GCD}\left(p, v_{1}, v_{2}, \ldots\right)=1\right)$. This solution generates other solutions in the form of a cycle

$$
y_{i}(X)=a_{1} \varepsilon^{v_{1} i} X^{v_{1} / p}+a_{2} \varepsilon^{v_{2} i} X^{v_{2} / p}+\cdots, \quad i=0, \ldots, p-1,
$$

where $\varepsilon$ is a primitive root of unity of degree $p$. Let $\theta_{j}=v_{j} / p$. Let us consider a sequence of tracks of the Newton algorithm for $f$ constructed from solution (10): $\varphi_{0}(X)=0, \ldots, \varphi_{j}(X)=a_{1} X^{\theta_{1}}+\cdots+a_{j} X^{\theta_{j}}, \ldots$ Let us put $f^{(j)}:=f_{\varphi_{j-1}}$ and $\mathscr{N}^{(j)}:=\mathscr{N}_{\varphi_{j-1}}$. It is convenient to denote $v_{0}=p, \theta_{0}=0$. We have

PROPERTY 5.1.

(i) Polygon $\mathscr{N}^{(j)}$ consists of one face $S^{(j)}$, with inclination $v_{j} / p$, which touches the horizontal axis.

(ii) $\operatorname{deg}_{Y} \operatorname{in}\left(f^{(j)}, S^{(j)}\right)=\operatorname{GCD}\left(v_{0}, \ldots, v_{j-1}\right)$ for $j=1,2, \ldots$

(iii) Every root of $\operatorname{in}\left(f^{(j)}, S^{(j)}\right)$ has the multiplicity $\operatorname{GCD}\left(v_{0}, \ldots, v_{j}\right)$.

(iv) $t\left(f^{(j)}, S^{(j)}\right)=\frac{\operatorname{GCD}\left(v_{0}, \ldots, v_{j-1}\right)}{\operatorname{GCD}\left(v_{0}, \ldots, v_{j}\right)}$, 
$\begin{array}{ll}\text { (v) } & \alpha\left(S^{(j)}\right)=\sum_{j^{\prime}=1}^{j}\left(\operatorname{GCD}\left(v_{0}, \ldots, v_{j^{\prime}-1}\right)-\operatorname{GCD}\left(v_{0}, \ldots, v_{j^{\prime}}\right)\right) \frac{v_{j^{\prime}}}{p}+ \\ & \operatorname{GCD}\left(v_{0}, \ldots, v_{j}\right) \frac{v_{j}}{p} .\end{array}$

Recall the definition of a generalized characteristic $\left(b_{0}, \ldots, b_{h}\right)$ :

$$
\begin{aligned}
b_{0}=v_{0}, \ldots, b_{k} & =\min \left\{v_{j}>b_{k-1} \mid \operatorname{GCD}\left(v_{0}, \ldots, v_{j-1}\right)>\operatorname{GCD}\left(v_{0}, \ldots, v_{j}\right)\right\}, \\
\ldots, b_{h} & =\min \left\{v_{j} \mid \operatorname{GCD}\left(v_{0}, \ldots, v_{j}\right)=1\right\} .
\end{aligned}
$$

If $p=v_{0} \leq v_{1}$, then the generalized characteristic coincides with the topological characteristic $(\mathbf{k}=\mathbf{C})$. We call $b_{1} / p, \ldots, b_{h} / p$ characteristic exponents. From Property 5.1 we obtain

Corollary 5.2. $t\left(f^{(j)}, S^{(j)}\right)>1$ if and only if $v_{j} / p$ is a characteristic exponent.

By $j_{1}, \ldots, j_{h}$ let us denote the values of index $j$ for characteristic exponents and let $\alpha_{k}=\alpha\left(S^{\left(j_{k}\right)}\right), k=1, \ldots, h$. Furthermore let

$$
e_{k}=\mathrm{GCD}\left(v_{0}, \ldots, v_{j_{k}}\right)=\mathrm{GCD}\left(b_{0}, \ldots, b_{k}\right) \text {. }
$$

We have

COROLlary 5.3. All polar quotients obtained on the tracks of solution (10) have the form

$$
\alpha_{k}=\sum_{k^{\prime}=1}^{k}\left(e_{k^{\prime}-1}-e_{k^{\prime}}\right) \frac{b_{k^{\prime}}}{p}+e_{k} \frac{b_{k}}{p}, \quad k=1, \ldots, h,
$$

and appear with multiplicity $\left(e_{k-1} / e_{k}\right)-1$.

Let $n_{k}=e_{k-1} / e_{k}$. To take into consideration the multiplicity deriving from the remaining tracks, let us write cycle (11) in the form

$$
\begin{aligned}
y_{i}(X)= & \psi_{0}(X)+\psi_{1}\left(\varepsilon_{1}^{i_{1}} X^{1 / n_{1}}\right)+\psi_{2}\left(\varepsilon_{2}^{i_{1}+n_{1} j_{2}} X^{1 /\left(n_{1} n_{2}\right)}\right) \\
& +\cdots+\psi_{h}\left(\varepsilon_{h}^{i_{1}+n_{1} i_{2}+\cdots+n_{1} n_{2} \cdots n_{h-1} i_{h}} X^{1 /\left(n_{1} n_{2} \cdots n_{h}\right)}\right),
\end{aligned}
$$

where $\psi_{0}, \ldots, \psi_{h-1} \in \mathbf{k}[X], \psi_{h} \in \mathbf{k}[[X]], \varepsilon_{h}=\varepsilon, \varepsilon_{k}=\varepsilon^{n_{k+1} \cdots n_{h}}(k=1, \ldots, h-1)$ and $i=i_{1}+n_{1} i_{2}+\cdots+n_{1} n_{2} \cdots n_{h-1} i_{h}$ is the unique decomposition of $i \in\{0, \ldots$, $p-1\}$ such that $0 \leq i_{1} \leq n_{1}-1, \ldots, 0 \leq i_{h} \leq n_{h}-1$. Therefore the track

$$
\varphi_{j_{k}}(X)=\psi_{0}(X)+\psi_{1}\left(X^{1 / n_{1}}\right)+\cdots+\psi_{k-1}\left(X^{1 /\left(n_{1} \cdots n_{k-1}\right)}\right)
$$


generates the cycle composed of $n_{1} \cdots n_{k-1}$ elements. Hence the multiplicity of polar quotient $\alpha_{k}$ is

$$
n_{1} \cdots n_{k-1}\left(n_{k}-1\right)=\frac{e_{0}}{e_{1}} \cdots \frac{e_{k-2}}{e_{k-1}}\left(\frac{e_{k-1}}{e_{k}}-1\right)=\frac{e_{0}}{e_{k}}-\frac{e_{0}}{e_{k-1}} .
$$

Commentary. From Property 5.1 result the common relations between faces $S^{(j)}$ and $S^{(j+1)}$ depending whether or not $j$ stands in the characteristic position. If $j \notin\left\{j_{1}, \ldots, j_{h}\right\}$, then $\operatorname{GCD}\left(v_{0}, \ldots, v_{j-1}\right)=\operatorname{GCD}\left(v_{0}, \ldots, v_{j}\right)$ and faces $S^{(j)}, S^{(j+1)}$ have a common upper end.

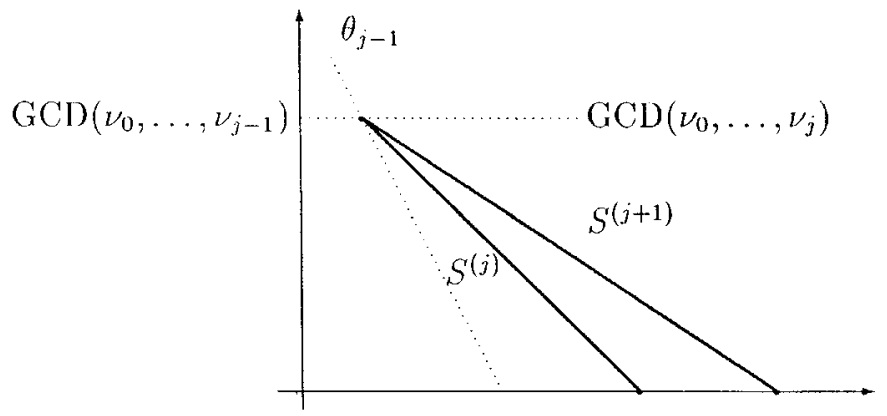

This means that polygon $\mathscr{N}^{(j+1)}$ does not contain a segment parallel to $S^{(j)}$. If $j=j_{k}$, then $\operatorname{GCD}\left(v_{0}, \ldots, v_{j-1}\right)=e_{k-1}>e_{k}=\operatorname{GCD}\left(v_{0}, \ldots, v_{j}\right)$.

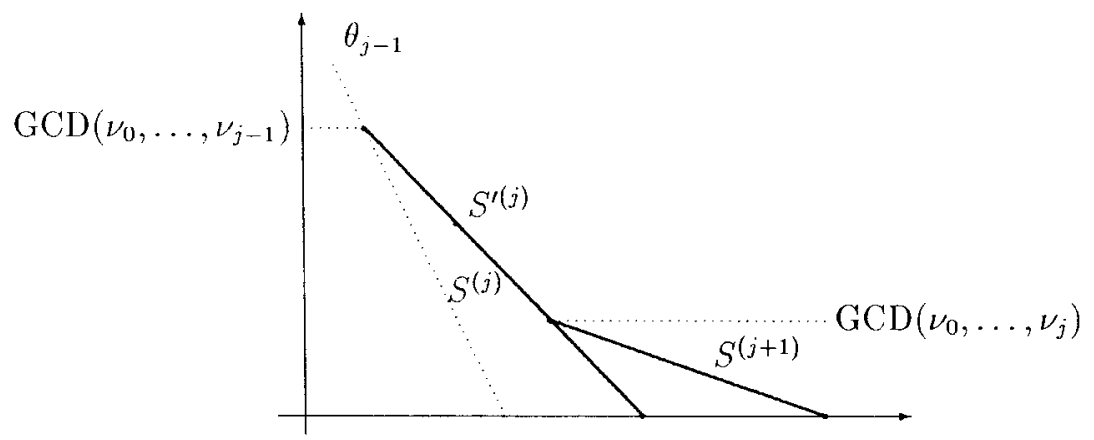

Let us divide face $S^{(j)}$ into $n_{k}=e_{k-1} / e_{k}$ equal parts. Face $S^{(j+1)}$ appears in place of the lowest part. Polygon $\mathscr{N}^{(j+1)}$ contains a face $S^{(j)}$ that is parallel to $S^{(j)}$. The length of the projection of $S^{(j)}$ onto the vertical axis is

$$
\operatorname{GCD}\left(v_{0}, \ldots, v_{j-1}\right)-\operatorname{GCD}\left(v_{0}, \ldots, v_{j}\right)=e_{k-1}-e_{k} .
$$

The sequence of polygons $\mathscr{N}^{(1)}, \mathscr{N}^{(2)}, \ldots$ approaches the polygon of series $f(X, y(X)+Y)$, which is composed of segments $S^{\prime\left(j_{1}\right)}, \ldots, S^{\prime\left(j_{h}\right)}$. 


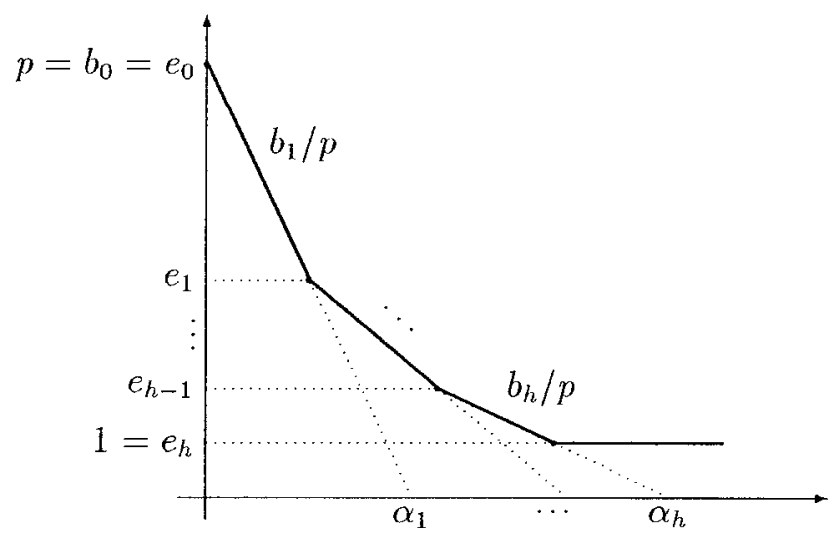

The numbers $\alpha_{1}, \ldots, \alpha_{h}$ represent the abscissae of the points where the lines determined by the faces intersect the horizontal axis (see: [GP1]).

Acknowledgements. The preliminary version of this paper emerged during the author's stay at the University of Bordeaux 1. The author wishes to express his thanks to Pierrette Cassou-Noguès for her invitation and hospitality as well as for many stimulating conversations.

\section{REFERENCES}

[CA $]$ E. Casas-Alvero, Singularities of plane curves, Cambridge Univ. Press, London Math. Soc., Lecture Notes, Series 276 (2000), xvi+345pp.

[Can] J. Cano, The Puiseux theorem for differential equations, in D. T. Lê, K. Saito, B. Teissier (eds), Singularity Theory, Word Scientific Publ., 1995, 128-152.

[D] F. Delgado De la Mata, A factorization theorem for the polar of a curve with two branches, Compositio Math. 92 (1994), 327-375.

[E] H. EgGers, Polarinvarianten und die Topologie von Kurvensingularitaten, Bonner Mathematische Schriften, 147, 1983.

[Eph] R. Ephraim, Special polars and curves with one place at infinity, Proc. of Symp. in Pure Math. vol. 40, Part I, 1983, 353-359.

[Ga] E. García-Barroso, Sur les courbes polaires d'une courbes plane réduite, Proc. of the London Math. Soc. (3) 81 (2000), 1-28.

[GP1] J. Gwoździewicz, A. Ploski, On the Merle formula for polar invariants, Bull. Soc. Sci. Letters Łódź 41(7), 1991, 61-67.

[GP2] J. GwoźDziewicz, A. PŁoski, On the polar quotients of an analytic plane curve, Kodai Math. J., 25(1) (2002), 43-53.

[KP] T. C. Kuo AND A. Parusiński, Newton polygon relative to an arc, Eds. J. W. Bruce, F. Tari, Real and Complex Singularities, São Carlos (1998), 76-93.

[L1] A. LenarciK, On the Lojasiewicz exponent of the gradient of a holomorphic function, in Singularities Symposium - Lojasiewicz 70, Banach Center Publ. 44 Inst. Math., Polish Acad. Sci., Warszawa, 1998, 149-166.

[L2] A. LenarciK, Newton algorithm and Puiseux series, Proceedings of the XXIV Conference of Analytic and Algebraic Geometry, Łódź 2003, 22-32 (in Polish, French version exists). 
POLAR QUOTIENTS OF A PLANE CURVE AND THE NEWTON ALGORITHM 353

[LP] A. Lenarcik, A. Ploski, Polar invariants of plane curves and the Newton polygon, Kodai Math. J. 23 (2000), 309-319.

[LMP] A. Lenarcik, M. Masternak, A. Ploski, Factorisation of the polar curve and the Newton polygon, Kodai Math. J., 26(3) (2003), 288-303.

[LMW1] Lê Dung Trang, F. Michel, C. Weber, Sur le comportement des polaires associées aux germes de courbes planes, Compositio Math., 72 (1989), 87-113.

[LMW2] Lê Dung Trang, F. Michel, C. Weber, Courbes polaires et topologie des courbes planes, Ann. scient. Éc. Norm. Sup. $4^{e}$ série, t. 24, 1991, 141-169.

[M] M. MerLe, Invariant polaires des courbes planes, Invent. Math. 41 (1977), 103-111.

[T] B. TeIsSIER, Variétés polaires, Invent. Math. 40 (1977), 267-292.

[W] R. Walker, Algebraic curves, Princeton University Press 1950.

[Wh] H. Whitney, Complex Analytic Varieties, Addison-Wesley, Reading, (1972).

Department of Mathematics, Technical University

AL. 1000 L PP 7, 25-314 Kielce, Poland

E-mail: ztpal@tu.kielce.pl 